\title{
On the Relationship between Field Amplitude Distribution, Its Maxima Distribution, and Field Uniformity inside a Mode-Stirred Reverberation Chamber
}

\author{
M. A. García-Fernández, C. Decroze, D. Carsenat, N. Arsalane, and G. Andrieu \\ OSA Department, XLim Laboratory, UMR CNRS 6172, University of Limoges, 123 avenue Albert Thomas, \\ 87060 Limoges Cedex, France \\ Correspondence should be addressed to M. A. García-Fernández, garciafernandez.ma@upct.es
}

Received 2 December 2011; Accepted 24 January 2012

Academic Editor: David A. Sanchez-Hernandez

Copyright (C 2012 M. A. García-Fernández et al. This is an open access article distributed under the Creative Commons Attribution License, which permits unrestricted use, distribution, and reproduction in any medium, provided the original work is properly cited.

\begin{abstract}
A mode-stirred reverberation chamber (RC) is nowadays a commonly accepted performing tool for over-the-air (OTA) communication system evaluation, and their standardization is underway. Before performing active measurements of wireless communication systems using an RC, field uniformity inside the RC working volume has to be measured following the calibration method described in IEC standards 61000-4-21 and 61000-4-3, which requires 24 calibration measurements of field amplitude. In this contribution, we present the statistical laws that describe electromagnetic field maxima distribution, and based on them, a novel expression that could be useful to obtain a lower limit for the number of stirrer positions required at least to obtain a specific value for the normalized dispersion used to evaluate field uniformity with the IEC calibration method, being therefore of particular interest for OTA measurements.
\end{abstract}

\section{Introduction}

A mode-stirred reverberation chamber (RC) is an electrically large, highly conductive enclosed cavity commonly accepted as performing tool for electromagnetic (EM) measurements (both emissions and immunity) on electronic equipment and for over-the-air (OTA) communication system evaluation. It is typically equipped with mechanical stirrers that modify its electromagnetic field boundary conditions, and when it is well stirred, that is, when a sufficient number of modes are excited, the resulting environment is essentially statistically uniform and statistically isotropic (i.e., the energy having arrived from all aspect angles and at all polarizations) with independence of location [1], achieving the field uniformity requirements, except for those observation points in close proximity to walls [2] and nearby objects. The field uniformity property of an ideal reverberation chamber is such that the mean-square value of the electric field and its rectangular components is considered independent of position [1]; likewise, the real and imaginary parts of each rectangular component of the electric and magnetic field throughout the chamber are Gaussian distributed, independent with identical variances; thus, the electric or magnetic field inside an ideal RC follows a single-cluster Rayleigh probability density function in amplitude and uniform distribution of phase, whereas the power fits an exponential one [3], which resembles the multipath fading in indoor scenarios of wireless communications systems. This RC behavior concerns high frequencies and can be qualified of "asymptotic" or "overmoded." On another hand, the "undermoded" case corresponds to the lower part of the spectrum that is close to the lowest usable frequency (LUF) $[4,5]$. Standard guidelines for RC operation usually involve estimating the LUF from the field uniformity of a given working volume. Field uniformity also permits to assess the behavior goodness of the RC, and its definition is based on the calibration procedure $[4,5]$. The field is determined at eight points located at the working volume corners, as depicted in Figure 1, and 


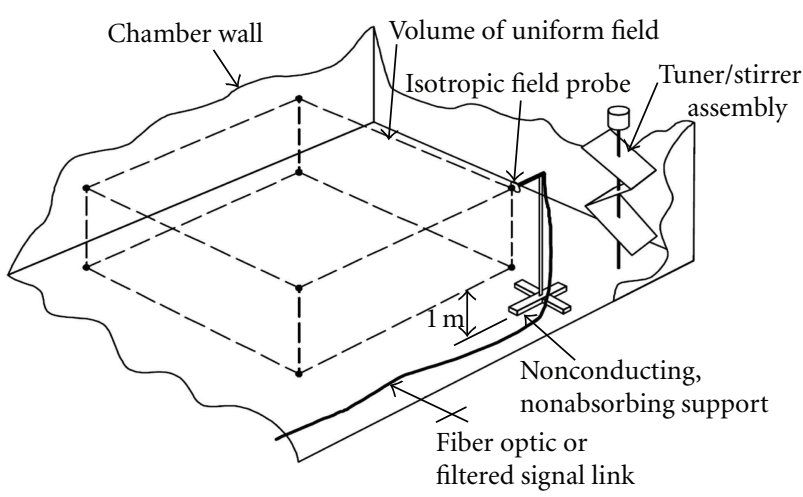

- Probe location for calibration

FIGURE 1: Chamber working volume [4].

normalized (to the square root of the input power). For each of the $8 \times 3$ normalized rectangular components, $E_{R}$, the maximum value among $N^{*}$ stirrer positions, $E_{R \text { Max }}$, is evaluated. From these $E_{R \text { Max }}$ maxima values, the mean value $\left\langle E_{R \text { Max }}\right\rangle$ and empiric standard deviation $\sigma_{E_{R M a x}}$ are calculated. Then, field uniformity is evaluated by calculating a normalized dispersion, $S(\mathrm{~dB})$, expressing the standard deviation $\sigma_{E_{R M a x}}$ in terms of $\mathrm{dB}$ relative to the mean $\left\langle E_{R \text { Max }}\right\rangle$, that is,

$$
S(\mathrm{~dB})=20 \log _{10}\left(1+\frac{\sigma_{E_{R M a x}}}{\left\langle E_{R \operatorname{Max}}\right\rangle}\right) .
$$

This is typically the figure of merit used to assess the performance of the RC, since the field within it is considered to be uniform if $S(\mathrm{~dB})$ is within $3 \mathrm{~dB}$ above $400 \mathrm{MHz}$, $4 \mathrm{~dB}$ at $100 \mathrm{MHz}$ decreasing linearly to $3 \mathrm{~dB}$ at $400 \mathrm{MHz}$, and within $4 \mathrm{~dB}$ below $100 \mathrm{MHz}$ [4]. Thus, field uniformity is a requirement for an $\mathrm{RC}$ to become a site for electromagnetic compatibility tests, in both radiated emission and susceptibility, and to provide measure reproducibility. Once field uniformity is attained in the working volume defined inside an RC, if a specific lower value of $S(\mathrm{~dB})$ is desired in order to obtain more accuracy for certain measurements, a higher number $N^{*}$ of stirrer positions are required, and if it is determined only through trial and error, calibration costs will increase, owing to the fact that calibration according to IEC standards $[4,6]$ is a long process, as explained before. Thus, in this contribution, we present the statistical laws that describe electromagnetic field maxima distribution, and based on them, we develop a novel methodology which relates the normalized dispersion $S(\mathrm{~dB})$ and the number $N$ of independent samples among the ones given by $N^{*}$ stirrer positions (whose relationship is estimated in [7]) when uniformity of the field amplitude distribution of the 3 rectangular components at the 8 points of the working volume defined inside an RC is accomplished. This methodology does not replace the IEC calibration method $[4,6]$, since the only way to ensure the required uniformity is to perform the corresponding 24 measurements for $N^{*}$ stirrer positions, and check that the collected samples are identically distributed, fitting a certain asymptotic law whose cumulative distribution function (CDF) is known. But once this is accomplished, the presented methodology can help to search for the number $N^{*}$ of stirrer positions necessary to obtain a desired lower $S(\mathrm{~dB})$ value quicker than only through trial and error, and thus decreasing calibration costs. This methodology has been validated through both simulations (by a Monte Carlo simulation, following the work presented in [5]) and measurements inside an RC. This contribution is of special interest for OTA measurements, whose standardization is underway [8], since it gives insights on the relationship between field amplitude distribution, its maxima distribution, and under the previously mentioned conditions, field uniformity inside an RC.

\section{Statistical Derivation of Field Amplitude, Maxima Distribution, and Its Relationship with Field Uniformity}

As in [9], where extreme value theory is used to deal with asymptotic distributions of extreme values, such as maxima, let $X_{1}, X_{2}, \ldots, X_{N}$ be independent and identically distributed (i.i.d.) random variables with a parent distribution function $F(x)$ and maxima $Y_{N}=\max _{1 \leq i \leq N}\left\{X_{i}\right\}$. If there exist constants $a_{N} \in \mathbb{R}, b_{N}>0$, and some nondegenerate distribution function $G$ such that the distribution of $\left(Y_{N}-\right.$ $\left.a_{N}\right) / b_{N}$ converges to $G$, then $G$ belongs to one of the three standard extreme value distributions: Frechet, Webull, and Gumbel distributions. The following lemma indicates a sufficient condition for a parent distribution function $F(x)$ belonging to the domain of attraction of the Gumbel distribution.

Lemma 1. Let $X_{1}, X_{2}, \ldots, X_{N}$ be i.i.d. random variables with a parent distribution function $F(x)$. Define $\omega(F)=\sup \{x$ : $F(x)<1\}$. Assume that there is a real number $x_{1}$ such that, for all $x_{1} \leq x<\omega(F), f(x)=F^{\prime}(x)$ and $F^{\prime \prime}(x)$ exist and $f(x) \neq 0$. If

$$
\lim _{x \rightarrow \omega(F)} \frac{d}{d x}\left[\frac{1-F(x)}{f(x)}\right]=0,
$$

then there exist constants $a_{N}$ and $b_{N}>0$ such that ( $Y_{N}-$ $\left.a_{N}\right) / b_{N}$ uniformly converges in distribution to a normalized Gumbel random variable as $N \rightarrow \infty$. The normalizing constants $a_{N}$ and $b_{N}$ are determined by

$$
\begin{gathered}
a_{N}=F^{-1}\left(1-\frac{1}{N}\right), \\
b_{N}=F^{-1}\left(1-\frac{1}{N e}\right)-F^{-1}\left(1-\frac{1}{N}\right),
\end{gathered}
$$

where $F^{-1}(x)=\inf \{y: F(y) \geq x\}$ [9-11]. Therefore, if the amplitude of an electric field rectangular component inside the $R C$ working volume, $E_{R}$, follows a parent distribution function $F(x)$, then the maxima values among $N$ independent samples obtained through the stirring process, $E_{R \text { Max }}$, will follow a 
Gumbel distribution, whose probability density function (PDF) is

$$
\begin{aligned}
g\left(y ; a_{N}, b_{N}\right)= & \frac{1}{b_{N}} \cdot \exp \left[-\frac{y-a_{N}}{b_{N}}\right] \\
& \cdot \exp \left[-\exp \left[-\frac{y-a_{N}}{b_{N}}\right]\right]
\end{aligned}
$$

[12], where its location and scale parameters, $a_{N}$ and $b_{N}$, respectively, can be straightforwardly derived from (3). For example, if the amplitude of an electric field rectangular component inside an $R C$ is Rayleigh distributed, that is, $E_{R} \sim$ Rayleigh $(\sigma)$, since the assumption in (2) is accomplished for $F(x)=$ Rayleigh $(\sigma)$, the maximum value among $N^{*}$ stirrer positions will be asymptotically Gumbel distributed, that is, $E_{R \operatorname{Max}} \sim \operatorname{Gumbel}\left(a_{N}, b_{N}\right)$, with $a_{N}=\sqrt{2 \sigma^{2} \ln (N)}$ and $b_{N}=\sqrt{2 \sigma^{2}}(\sqrt{1+\ln (N)}-\sqrt{\ln (N))})$, where $N$ is the number of independent samples among the ones given by the $N^{*}$ stirrer positions (whose relationship is estimated in [7], as mentioned in the introduction). Moreover, according to IEC 61000-4-21 standard [4], if the field amplitudes are normalized to the square root of the input power, $\sqrt{2 \sigma^{2}}$ will be equal to 1 , and so the expressions for $a_{N}$ and $b_{N}$ are even more simplified, that is, $a_{N}=\sqrt{\ln (N)}$ and $b_{N}=\sqrt{1+\ln (N)}-\sqrt{\ln (N) \text {. Fur- }}$ thermore, after knowing that the distribution of maxima values uniformly converges to a Gumbel one, in order to calculate its moments, moment convergence has to be ensured. Even though convergence in distribution is not equivalent to moment convergence in general, following the relation between convergence in distribution and moment convergence $[9,13]$, convergence in distribution for the maximum of nonnegative random variables, as field amplitude inside an $R C$, results in moment convergence. Thus, the mean value $\left\langle E_{R \text { Max }}\right\rangle$ and empiric standard deviation $\sigma_{E_{R M a x}}$ of maxima values $E_{R \text { Max }}$ which follow a Gumbel distribution can be straightforwardly derived from their mathematical definition, using the Gumbel $P D F$ described in (4), resulting in

$$
\begin{aligned}
\left\langle E_{R \operatorname{Max}}\right\rangle & =a_{N}+\gamma b_{N}, \\
\sigma_{E_{R \operatorname{Max}}} & =\frac{\pi}{\sqrt{6}} b_{N},
\end{aligned}
$$

where $\gamma=0.5772 \ldots$ is the Euler constant [11], and $a_{N}$ and $b_{N}$ were already presented in (3).

Likewise, in the particular case of attaining field uniformity inside an RC, evaluated through the IEC calibration method $[4,6]$, and when the collected samples of field amplitude are identically distributed for the 3 rectangular components at the 8 points of its working volume, field uniformity could also be evaluated by the following normalized dispersion:

$$
\widetilde{S}(\mathrm{~dB})=20 \log _{10}\left(1+\frac{\pi}{\sqrt{6}} \frac{1}{\gamma+\left(a_{N} / b_{N}\right)}\right) .
$$

It is obtained by substituting $\left\langle E_{R \text { Max }}\right\rangle$ and $\sigma_{E_{R M a x}}$ in (1) for the values obtained from (5). However, $\widetilde{S}(\mathrm{~dB})$ is not necessarily equal to $S(\mathrm{~dB})$, and the previously described distribution uniformity needs to be evaluated by the IEC calibration method $[4,6]$ and attained in order to be comparable. The advantage that (6) provides is that once the described distribution uniformity is accomplished, in order to achieve a lower dispersion $S(\mathrm{~dB})$, the higher number $N^{*}$ of stirrer positions which would be required at least can be calculated faster using it if we consider that the ratio between $N$ and $N^{*}$ should not be increased when $N^{*}$ is increased if the stirring method is not altered. It is worthy to note that field uniformity evaluated by means of (6) only depends on its coefficients $a_{N}$ and $b_{N}$, which are described in (3), and thus on the distribution of the field amplitude inside the RC working volume, $F(x)$, and the number of independent samples, $N$, among the ones given by the $N^{*}$ stirrer positions. Following the previous example, if the field amplitude distribution results to be Rayleigh $(\sigma)$ for the 3 rectangular components at the 8 points of the working volume defined inside an RC, (6) becomes

$$
\tilde{S}(\mathrm{~dB})=20 \log _{10}\left(1+\frac{\pi}{\sqrt{6}} \frac{1}{\gamma+1 /(\sqrt{1+(1 / \ln (N))}-1)}\right),
$$

which depends only on the number $N$ of independent collected samples. Thus, (6) and (7) can also be useful to calculate the number $N$ of independent samples among the ones given by the $N^{*}$ stirrer positions from the $S(\mathrm{~dB})$ value calculated according to IEC standards $[4,6]$ when the previously described distribution uniformity is accomplished.

\section{Validation through Monte Carlo Simulations}

Following the works presented in $[5,14]$, we use a Monte Carlo simulation in order to validate the expression presented in (5), and also the one in (6) for the case of having the previously described distribution uniformity, by confirming that the maxima values among $N$ independent samples which follow a given parent distribution $F(x)$ follow a distribution that converges asymptotically to a Gumbel one, comparing the maxima mean value and standard deviation to the ones given by (5), and also comparing the field uniformity dispersion $S(\mathrm{~dB})$ to the $\widetilde{S}(\mathrm{~dB})$ given by (6).

Thus, using a MATLAB script, 24 signals of a determined length $N$ (modeling the 3 rectangular components of the electric field at the 8 working volume corners along the same number of independent stirrer positions) are randomly generated following one specific distribution function, $F(x)$. Then, the maximum value of each signal is calculated, and the mean value and standard deviation of these 24 maxima are computed. The procedure is iterated 1000 times, and the averaged results are depicted in Figures 2 and 3, in solid lines. They are compared with the ones predicted by (5), in dotted lines. Different numbers $N$ of independent samples (or alternatively, independent stirrer positions), and a Rice distribution as parent distribution, with different $K$-factors (including $K=0$ in order to study the results for a Rayleigh distribution) have been used. As we can see, since maxima distribution only converges asymptotically to a Gumbel one (but does not fit exactly for a finite number $N$ of independent 


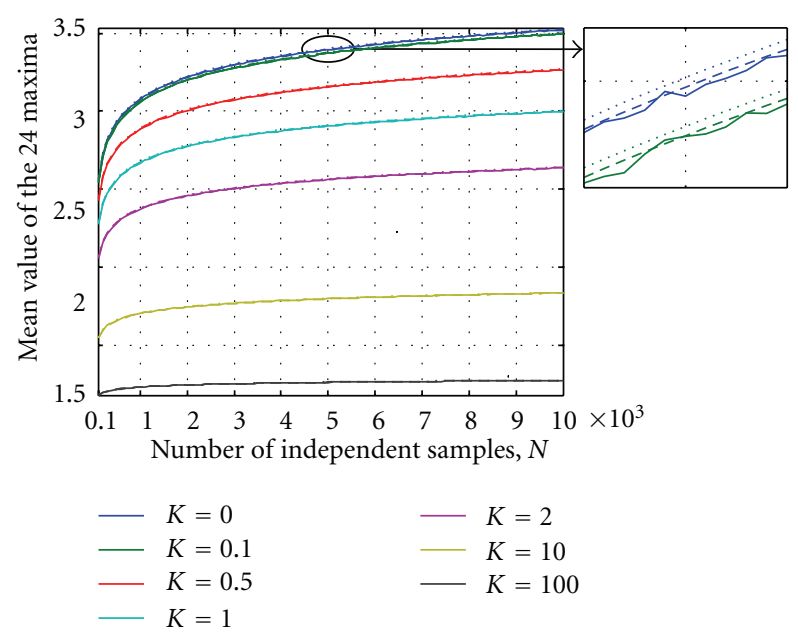

Figure 2: Mean value of the 24 maxima, averaged along 1000 runs of a Monte Carlo simulation, and the comparison to the values predicted by (5), for a Rice distribution as parent distribution with different $K$-factors.

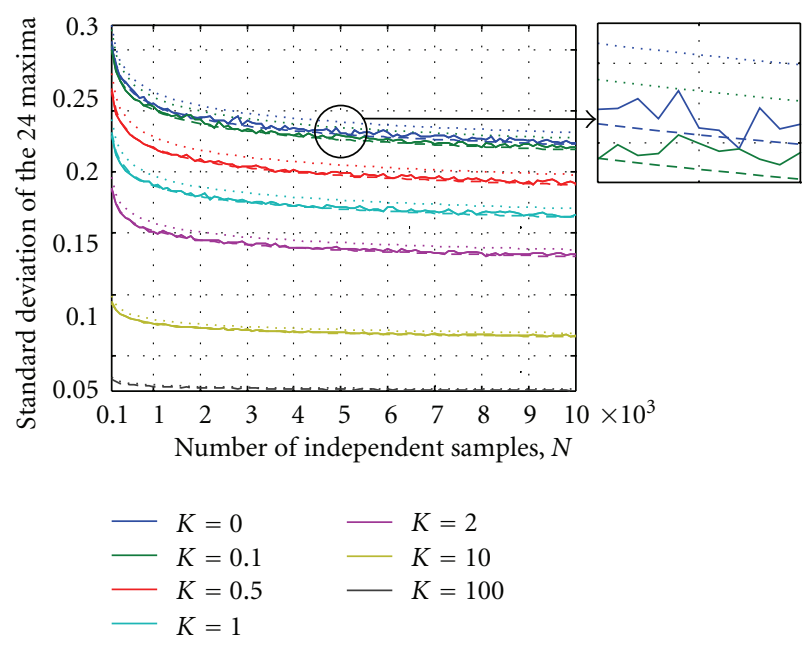

Figure 3: Standard deviation of the 24 maxima, averaged along 1000 runs of a Monte Carlo simulation, and the comparison to the values predicted by (5), for a Rice distribution as parent distribution with different $K$-factors.

samples), there is a small bias from the simulated results to the predicted ones. However, this bias has also been corrected by multiplying $b_{N}$, obtained following (3), by a factor of $23 / 24=0.9583 \ldots$ (related to the $M=24$ maxima, as $(M-1) / M)$, and recalculating the statistics by means of (5), as shown in dashed lines.

Afterwards, field uniformity is evaluated by calculating the normalized dispersion $S(\mathrm{~dB})$ according to IEC standards $[4,6]$ from the simulated samples (using the statistics of the 24 maxima), and the obtained values are averaged over the 1000 Monte Carlo runs and depicted (in green) in Figures $4-6$, for different $K$-factors. Moreover, $S(\mathrm{~dB})$ extrema $\left[S(\mathrm{~dB})_{\min } S(\mathrm{~dB})_{\max }\right]$ have been also computed, as in [5] (and depicted in blue and red, resp.). These results are

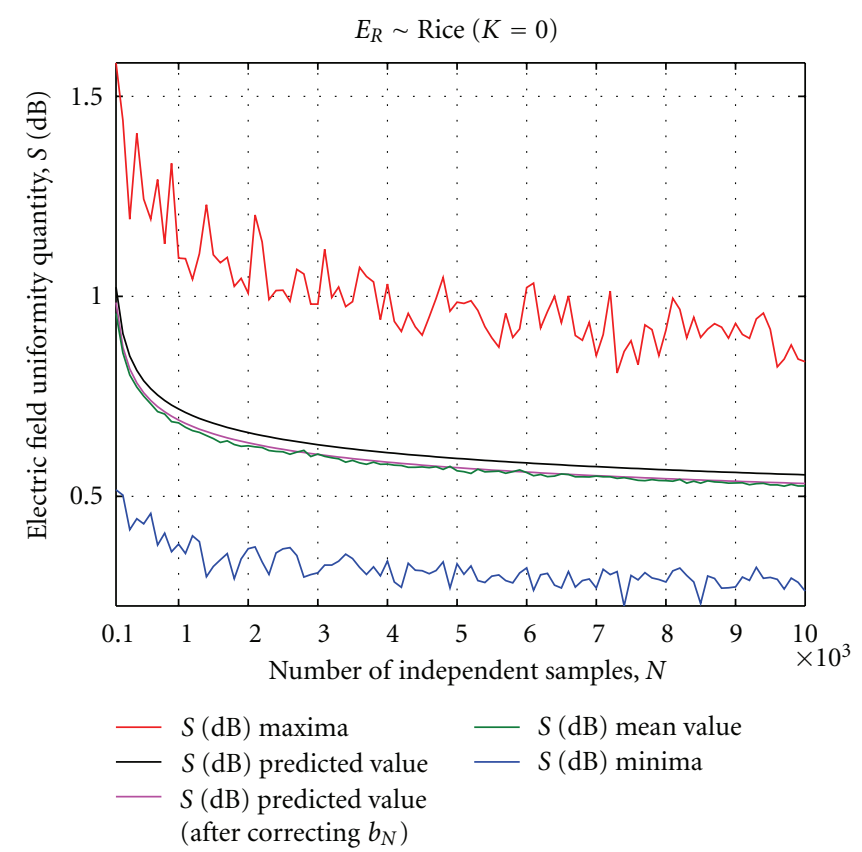

FIgURE 4: $S(\mathrm{~dB})$ mean value, averaged along 1000 runs of a Monte Carlo simulation, and the comparison to the values predicted by (6), for a Rayleigh distribution as parent distribution (Rice distribution with $K=0$ ).

compared with the ones predicted by $(6), \widetilde{S}(\mathrm{~dB})$ (depicted in black). Since a small bias appears for the same reason already explained for the statistics (mean value and standard deviation) case, $b_{N}$ is multiplied by the same correcting factor of $23 / 24$, and the resulting $\widetilde{S}(\mathrm{~dB})$ is also presented (in magenta).

As we can see, the mean value of the calculated $S(\mathrm{~dB})$, averaged along the 1000 runs of the Monte Carlo simulation, asymptotically converges to the $\tilde{S}$ (dB) given by (6), with $b_{N}$ calculated following (3). Moreover, after multiplying $b_{N}$ by the correction factor of $23 / 24$, the mean value of the calculated $S(\mathrm{~dB})$ fits $\widetilde{S}(\mathrm{~dB})$. Similar results are obtained for other parent distributions, $F(x)$, as Weibull or Nakagami, and they are therefore not presented here for brevity. It is also worthy to note that field uniformity is improved when the $K$-factor of the Rice distribution (followed by the amplitude of the electric field rectangular components) increases. This is obvious since when the dominant direct coupling component of the field is increased, the Rice $K$-factor is also increased, and in this case, the coefficient of variation of amplitude of the electric field rectangular components is reduced, thus decreasing the normalized dispersion used to evaluate the field uniformity. Likewise, we can observe from the results that the absolute deviation between both extrema of $S(\mathrm{~dB})$ along the 1000 Monte Carlo simulations (which are performed considering distribution uniformity) is up to $1 \mathrm{~dB}$ (when each rectangular component is modeled by $N=100$ independent samples following a Rayleigh distribution), and this should be taken in consideration when an RC calibration is performed according to the IEC standards $[4,6]$ in order 


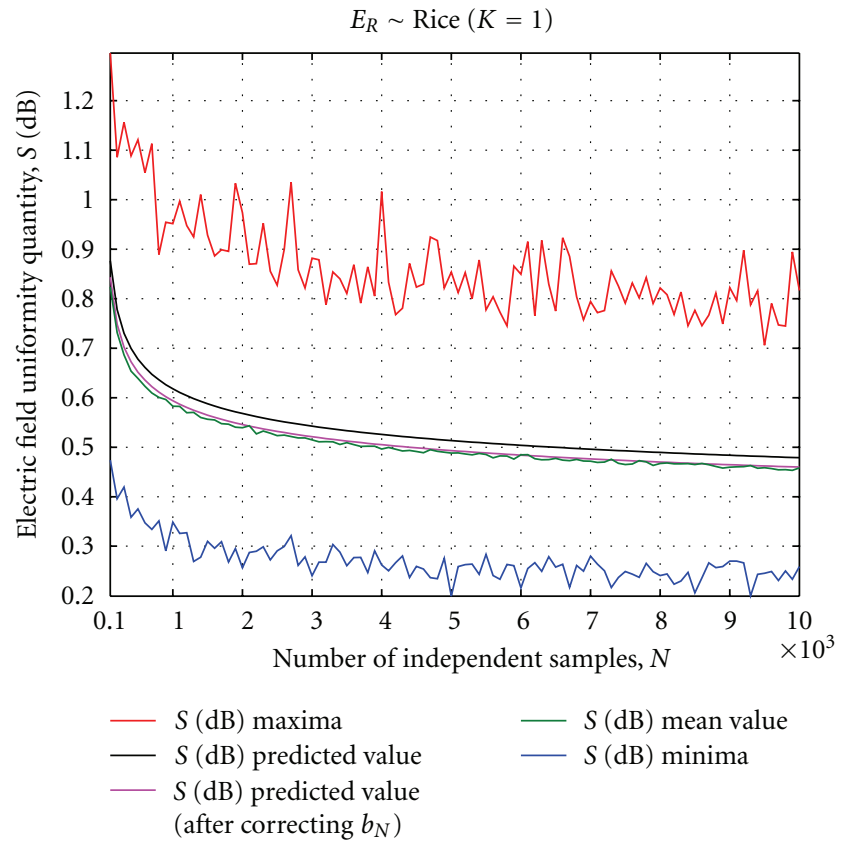

Figure 5: $S(\mathrm{~dB})$ mean value, averaged along 1000 runs of a Monte Carlo simulation, and the comparison to the values predicted by (6), for a Rice distribution with $K=1$ as parent distribution.

to consider the field within the $\mathrm{RC}$ as uniform or not, specially when the value of $S(\mathrm{~dB})$ resulted too close to the corresponding limit of $3 \mathrm{~dB}$ or $4 \mathrm{~dB}$ [4] (depending on the frequency, as mentioned in the introduction).

\section{Validation through Measurements in Reverberation Chambers}

In order to ensure the validity of (5), and also the one of (6) when the previously described distribution uniformity is accomplished, several measurements have been carried out in one of the RCs of the OSA Department at XLim Laboratory, whose inner view is shown in Figure 7.

Following the IEC calibration method $[4,6]$, the amplitude of the $x_{-}, y$-, and $z$-rectangular components of the electric field has been measured using a triaxial probe located at the 8 corners of a delimited working volume, at a frequency of $2 \mathrm{GHz}$. At this frequency, the $\mathrm{RC}$ is considered to work under the "overmoded" regime inside its factory-delimited working volume, and this requirement is necessary in order to compare the results obtained from the measurements to the ones provided by the equations presented before. The associated empirical CDFs are shown in Figure 8, considering two different working volumes: one slightly inside the factory-delimited working volume and the factory-delimited working volume itself. As we can see, they asymptotically converge to a Rayleigh distribution (the differences will be attributed to a lack of independent samples, since the measurement counts only with $N^{*}=100$ stirrer positions). Then, the maxima values for each one of the normalized rectangular components are evaluated,

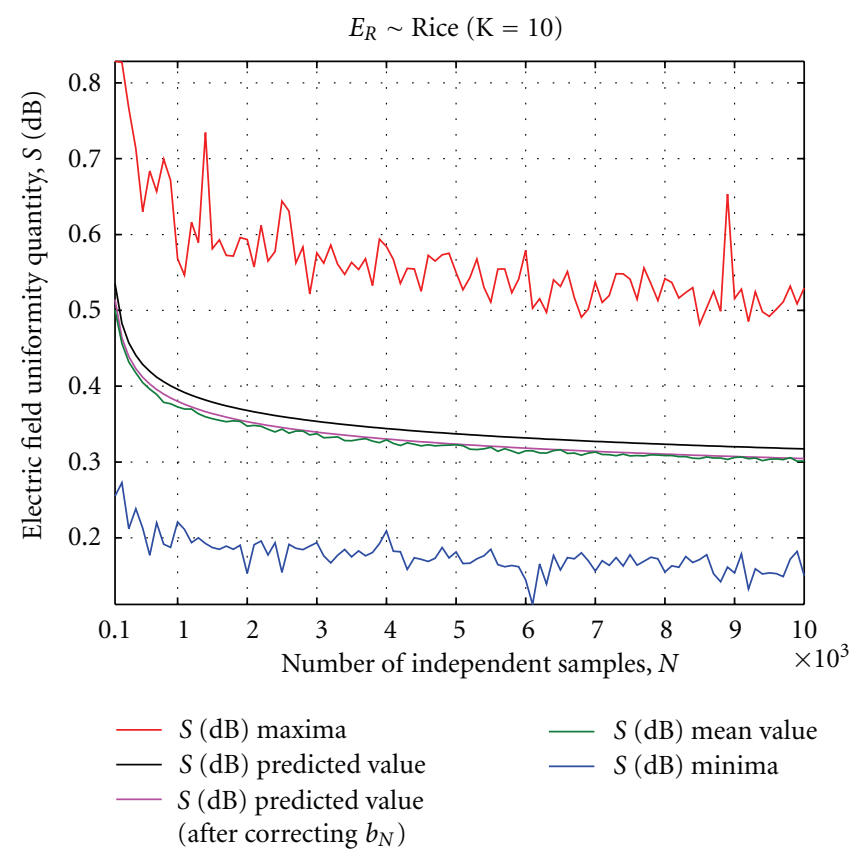

Figure 6: $S(\mathrm{~dB})$ mean value, averaged along 1000 runs of a Monte Carlo simulation, and the comparison to the values predicted by (6), for a Rice distribution with $K=10$ as parent distribution.

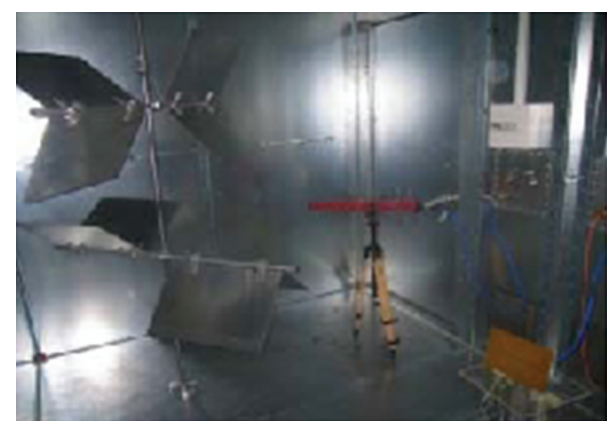

FIGURE 7: Inner view of the RC used in this study.

TABLE 1: Measured results.

\begin{tabular}{lccc}
\hline Measurement & $\left\langle E_{R \text { Max }}\right\rangle$ & $\sigma_{E_{R M a x}}$ & $S(\mathrm{~dB})$ \\
\hline I & 2.2625 & 0.3284 & 1.1771 \\
II & 2.2249 & 0.2830 & 1.0399 \\
\hline
\end{tabular}

and from these maxima, $E_{R \text { Max }}$, the mean value $\left\langle E_{R \text { Max }}\right\rangle$ and empiric standard deviation $\sigma_{E_{R M a x}}$ are calculated and compared to the values given by (5). Finally, field uniformity is evaluated by calculating the normalized dispersion $S(\mathrm{~dB})$ according to IEC standards $[4,6]$, and the obtained value is compared to the one given (6). The results obtained from the measurements performed for the two different working volumes, following the IEC calibration method $[4,6]$, are shown in Table 1.

Likewise, when the amplitude of the rectangular components is considered to be Rayleigh, (5) gives $\left\langle E_{R \text { Max }}\right\rangle=$ 2.2739 and $\sigma_{E_{R \operatorname{Max}}}=0.2842$, for $N=100$ independent 


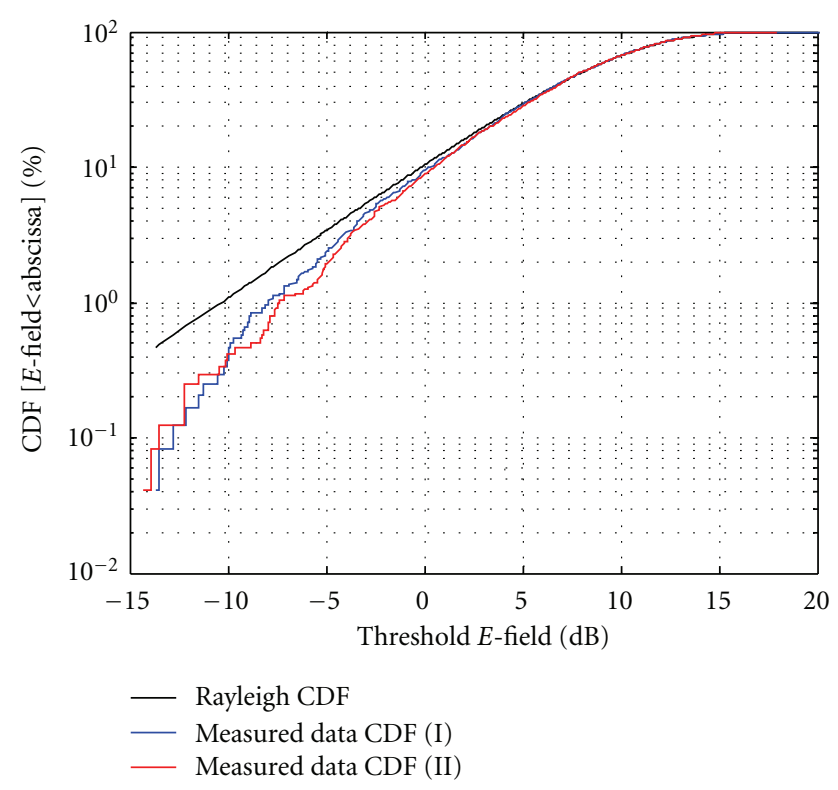

FIgURE 8: CDFs of the electric field $(x-, y$-, and $z$-rectangular components) at a frequency of $2 \mathrm{GHz}$.

samples. In addition, (6), or (7) since the considered distribution is a Rayleigh one, gives a value of $\widetilde{S}(\mathrm{~dB})=$ $1.0228 \ldots$, also for $N=100$ independent samples. As we can see, the measured values are very close to the one given by the equations presented in this contribution, since the assumption of having the same distribution for the amplitudes of all the rectangular components is accomplished for both measurements, as depicted in Figure 8. Moreover, the measured values of $S(\mathrm{~dB})$ resulted to be also situated between the minimum and maximum values, $\left[S(\mathrm{~dB})_{\min }=\right.$ $\left.0.5161, S(\mathrm{~dB})_{\max }=1.5841\right]$ calculated by the Monte Carlo simulation for $N=100$ independent samples, as previously depicted in Figure 4.

Furthermore, after performing the calibration according to the IEC standards $[4,6]$, and confirming that the distribution of the amplitude of the rectangular components of the electric field has resulted to be Rayleigh, if a lower $S(\mathrm{~dB})$ value was desired, in order to obtain a higher accuracy, the number $N^{*}$ of stirrer positions that would be required at least can be calculated using (7). For example, if $S(\mathrm{~dB})$ was desired to be lower than $0.5 \mathrm{~dB}$, substituting $\widetilde{S}(\mathrm{~dB})$ by $0.5 \mathrm{~dB}$ in (7), we obtain that the number $N$ of independent samples that are required at least for that purpose would be 29429 , and performing a calibration according to IEC standards $[4,6]$ with a number $N^{*}$ of stirrer positions lower than this higher number $N$ would be useless, since increasing the number $N^{*}$ of stirrer positions cannot decrease the distribution uniformity and cannot also increase the ratio between $N$ and $N^{*}$ if the stirring method is not altered. This is specially important after seeing that the $S(\mathrm{~dB})$ value obtained for $N^{*}=100$ stirrer positions is around $1 \mathrm{~dB}$, and that the absolute deviation from one calibration realization to another in the same conditions has resulted to be up to $1 \mathrm{~dB}$, as confirmed by the Monte Carlo simulations previously depicted in Figure 4 , and thus, the value of $S(\mathrm{~dB})=0.5 \mathrm{~dB}$ desired in this example could be obtained by chance using a lower number $N^{*}$ of stirrer positions but only because of a deviation, and not because the field is really as uniform as desired and as $S(\mathrm{~dB})=0.5 \mathrm{~dB}$ indicates. Therefore, (7), and in general, (6), becomes useful for calculating a lower limit for the number $N^{*}$ of stirrer positions to be selected in order to obtain a specific value for $S(\mathrm{~dB})$ dispersion when performing a calibration according to the IEC standards $[4,6]$, specially once distribution uniformity is attained in a previous calibration with a lower number $N^{*}$ of stirrer positions. In this way, the search for the desired value of $S(\mathrm{~dB})$ would be faster if compared to searching for it only through trial and error, and consequently, the associated calibration time and costs would be reduced.

\section{Discussion and Conclusions}

In this paper, the statistical laws that describe electromagnetic field maxima distribution as a Gumbel one with parameters given by (3) have been presented, thus permitting to calculate the statistics mean value and standard deviation of the maxima values distributed so using (5). Based on that, we present in (6) a novel expression which relates the normalized dispersion $S(\mathrm{~dB})$ used to evaluate field uniformity to the number $N$ of independent samples collected among the $N^{*}$ stirrer positions used to calibrate an RC according to the IEC standards $[4,6]$ for the special case of having distribution uniformity, that is, when the field amplitude of the 3 rectangular components measured at the 8 points of the working volume defined inside the RC follows the same distribution. This study has been successfully validated both through Monte Carlo simulations and measurements in an RC.

Moreover, as it has been shown in Figures 4-6, field uniformity measured through the IEC calibration method $[4,6]$ can vary more than $1 \mathrm{~dB}$ between two calibration realizations even when the amplitude of the electric field rectangular components is independent and identically distributed at the eight points located at the working volume corners. Thus, since repeating the calibration process enough times to calculate an estimation of the mean value of the normalized dispersion $S(\mathrm{~dB})$ is usually unaffordable due to time requirements, it is essential to know a lower limit for the number $N^{*}$ of stirrer positions to be selected in order to obtain a specific value for $S(\mathrm{~dB})$. This lower limit can be easily obtained from (6) by substituting $\widetilde{S}(\mathrm{~dB})$ by the desired $S(\mathrm{~dB})$ value, taking into account that the more distribution uniformity we have and the more independent the samples between two consecutive stirrer positions are, the more this lower limit approaches the real number $N^{*}$ of stirrer positions required for this purpose.

Therefore, this contribution is of special interest for OTA measurements, whose standardization is underway [8], since it not only gives insights on the relationship between field amplitude distribution and its maxima distribution, but also it could help to accelerate the achievement of a specific value of $S(\mathrm{~dB})$ when performing a calibration according to IEC 
standards $[4,6]$ compared to doing it only through trial and error and consequently could help to reduce calibration time and costs.

\section{Acknowledgment}

This research has been partially funded by a fellowship grant by Fundación Séneca, the Regional R\&D Coordinating Unit of the Region of Murcia (Spain).

\section{References}

[1] D. A. Hill, "Electromagnetic Theory of Reverberation Chambers," (US) Technical Note 1506, National Institute of Standards and Technology, 1998.

[2] L. R. Arnaut and P. D. West, "Electromagnetic reverberation near a perfectly conducting boundary," IEEE Transactions on Electromagnetic Compatibility, vol. 48, no. 2, pp. 359-371, 2006.

[3] D. A. Hill, "Plane wave integral representation for fields in reverberation chambers," IEEE Transactions on Electromagnetic Compatibility, vol. 40, no. 3, pp. 209-217, 1998.

[4] Electromagnetic Compatibility (EMC) - Part 4-21, "Testing and measurement techniques-reverberation chamber test methods," IEC 61000-4-21, 2003.

[5] G. Orjubin, E. Richalot, S. Mengué, and O. Picon, "Statistical model of an undermoded reverberation chamber," IEEE Transactions on Electromagnetic Compatibility, vol. 48, no. 1, pp. 248-251, 2006.

[6] Electromagnetic Compatibility (EMC)—Part 4-3, "Testing and measurement techniques_-radiated, radio-frequency, electromagnetic field immunity test," IEC 61000-4-3, 2006.

[7] C. Lemoine, P. Besnier, and M. Drissi, "Estimating the effective sample size to select independent measurements in a reverberation chamber," IEEE Transactions on Electromagnetic Compatibility, vol. 50, no. 2, pp. 227-236, 2008.

[8] M. A. García-Fernández, J. D. Sánchez-Heredia, A. M. Martínez-González, D. A. Sánchez-Hernández, and J. F. Valenzuela-Valdés, "Advances in mode-stirred reverberation chambers for wireless communication performance evaluation," IEEE Communications Magazine, vol. 49, no. 7, pp. 140-147, 2011.

[9] G. Song and Y. Li, "Asymptotic throughput analysis for channel-aware scheduling," IEEE Transactions on Communications, vol. 54, no. 10, pp. 1827-1834, 2006.

[10] H. A. David, Order Statistics, Wiley, New York, NY, USA, 1970.

[11] J. Galambos, The Asymptotic Theory of Extreme Order Statistics, Wiley, New York, NY, USA, 1978.

[12] E. J. Gumbel, "Statistical theory of extreme values and some practical applications," in A Series of Lectures, National Bureau of Standards, Applied Mathematics Series, 33, pp. 8-51, US Government Printing Office, Washington, DC, USA, 1954.

[13] J. Pickands III, "Moment convergence of sample extremes," The Annals of Mathematical Statistics, vol. 39, no. 3, pp. 881889, 1968.

[14] J. M. Ladbury, "Monte Carlo Simulation of Reverberation Chambers," Internal Note, National Institute of Standards and Technology (NIST), Boulder, Colo, USA, 1999. 

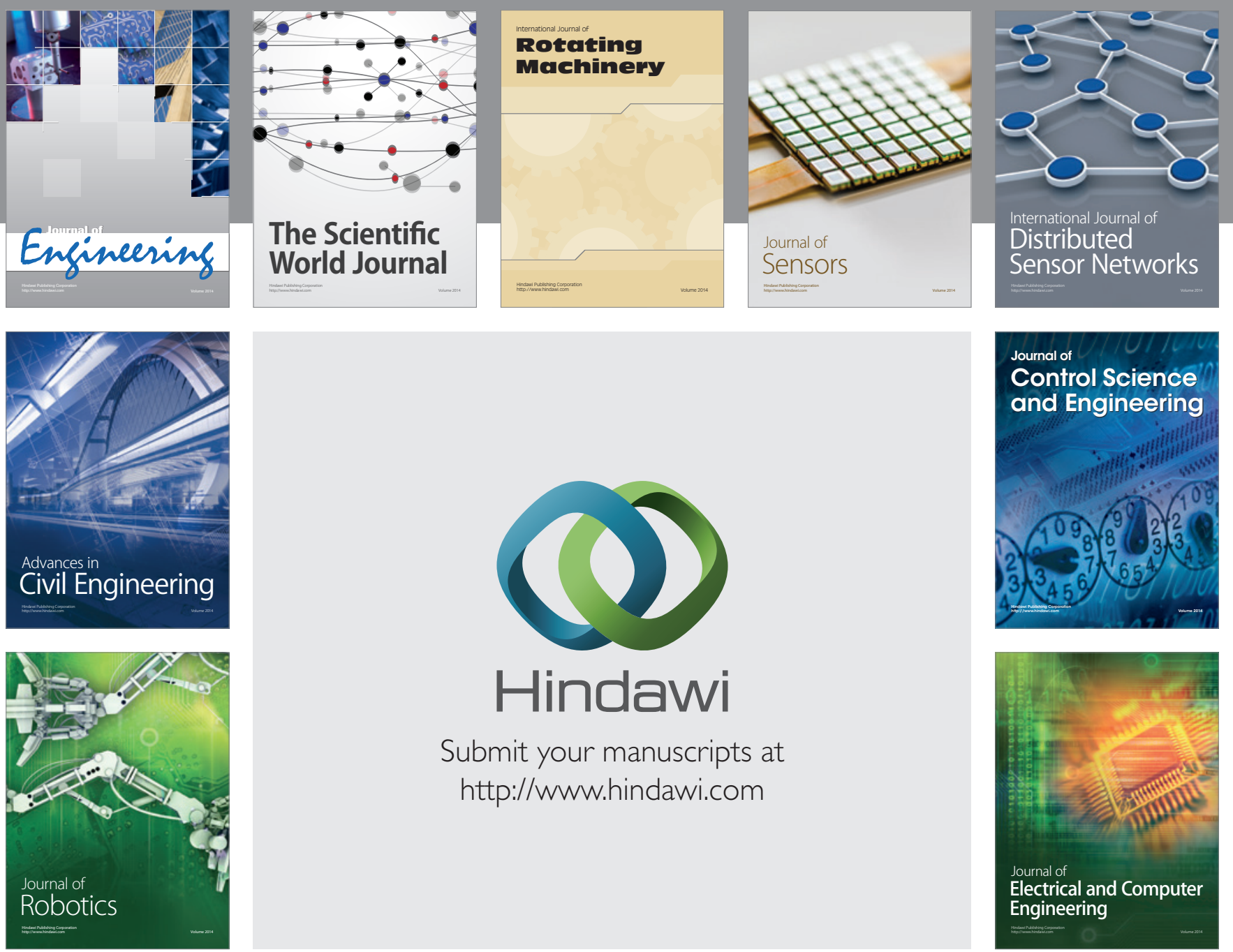

Submit your manuscripts at

http://www.hindawi.com
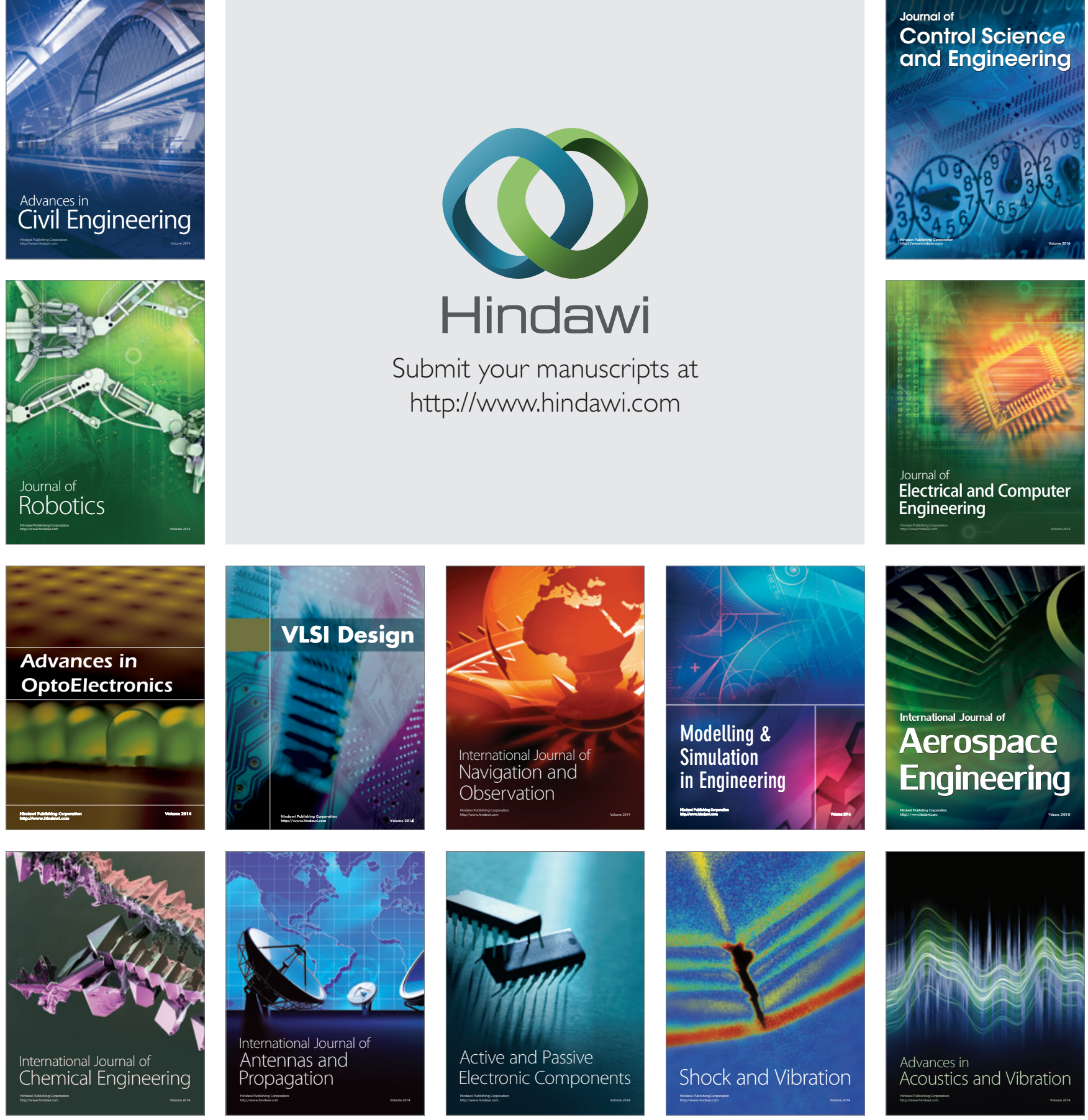